\title{
Un gène responsable d'insuffisance ovarienne prématurée est localisé sur le chromosome 3
}

Dans le numéro de janvier 1996 de médecine/sciences ( $m / s n^{\circ} 1$, vol. 12 , p. 108), l'un de nous faisait état d'un curieux syndrome où l'on observe à la fois des anomalies congénitales des paupières et une insuffisance ovarienne. Ce syndrome, connu sous le nom de blépharophimosis familial ou blépharophimosis-ptosis-épicanthus inversus (BPES) (MIM:110100), est une affection autosomique dominante caractérisée par une étroitesse majeure des fentes palpébrales pouvant entraîner une gêne importante de la vision. Deux formes particulières de ce syndrome ont été décrites. Le BPES de type I associe curieusement des malformations des paupières à une insuffisance ovarienne, alors que le BPES de type II ne donne que des anomalies oculaires. Dans les familles de type I, les femmes sont stériles; la transmission de l'affection ne se fait que par les hommes qui sont normalement fertiles. Dans les familles de type II, les femmes peuvent avoir des enfants sans problème et la transmission de la maladie se fait par les deux sexes [1]. Il est important de souligner que les 2 types de BPES ne se retrouvent jamais dans une même famille. Récemment, nous avons localisé un gène responsable du BPES type II sur le bras long du chromosome 3 en $3 q 22-q 23$ [2].

Ces résultats confirmaient, par une étude de liaison génétique, ce qui était fortement suspecté par les descriptions d'anomalies cytogénétiques intéressant toujours la bande 3q22q23 dans des cas sporadiques de BPES. Nous venons maintenant de montrer que la forme de BPES avec insuffisance ovarienne est située au même locus. Nous avons étudié trois familles indépendantes : une Italienne (P. Gasparini, San Giovanni Rotondo), une Israélienne (J. Zlotogora, Jérusalem) et une Française (M. Nicolino, Paris) regroupant un total de vingt individus BPES dont dix femmes stériles et six sujets sains. Nous avons obtenu un lod-score de 4,52 à $0 \%$ de recombinaison au locus D3S1316 permettant de localiser cette forme de BPES avec stérilité féminine en 3q22-q23 [3].

D'une façon remarquable la cause de la stérilité féminine diffère dans nos familles. Dans deux d'entre elles, les femmes ont une insuffisance ovarienne secondaire avec l'équivalent d'une ménopause précoce (entre 25 et $30 \mathrm{ans})$. Chez elles, la puberté et les premiers cycles menstruels se sont déroulés sans problème mais la fonction des ovaires est devenue rapidement déficitaire. Dans la troisième famille, au contraire, la fonction ovarienne n'a jamais été normale et il existe, dans ces cas, une dysgénésie gonadique féminine primitive [4]. A notre connaissance, il s'agit de la première localisation d'un gène autosomique impliqué à la fois dans le développement et dans le maintien de la fonction ovarienne. Ces deux fonctions étaient jusqu'à maintenant supposées être uniquement sous la dépendance de gènes localisés sur le chromosome X.

Deux hypothèses peuvent dès lors être formulées: soit le BPES de type I est un syndrome impliquant des gènes contigus ayant sous leur controle le développement de la paupière et de l'ovaire, soit des mutations différentes d'un seul et même gène sont responsables des deux phénotypes (anomalies des paupières avec ou sans insuffisance ovarienne).
Quoi qu'il en soit, la caractérisation du ou des gènes responsables de ce syndrome permettra de mieux appréhender à la fois le développement des annexes de l'œil et celui des gonades féminines.

P.A. J.-C.C. A.K. D.B. M.N.

1. Zlotogora J, Sagi M, Cohen T. The blepharophimosis, ptosis, and epicanthus inversus syndrome: delineation of two types. Am J Hum Genet $1983 ; 35: 1020-7$.

2. Amati P, Chomel JC, Nivelon-Chevalier A, Gilgenkrantz S, Kitzis A, Kaplan J, Bonneau D. A gene for blepharophimosis, ptosis, epicanthus inversus maps to chromosome 3q23. Hum Genet $1995 ; 96: 213-5$

3. Amati P, Gasparini P, Zlotogora J, Chomel JC, Kaplan J, Kitzis A, Bonneau D. A gene for premature ovarian failure and eyelid malformation maps to chromosome 3q22-q23. Am J Hum Genet (sous presse).

4. Nicolino M, Bost M, David M, Chaussain JL. Familial blepharophimosis: an uncommon marker of ovarian dysgenesis. I Ped Endocrinol Metabol $1995 ; 8: 127-33$. 